\title{
Production of spin-3 mesons in diffractive DIS
}

\section{F. Caporale}

Dipartimento di Fisica, Università della Calabria, and INFN - Gruppo Collegato di Cosenza, I-87036 Rende, Italy

E-mail: caporaledcs.infn.it

\section{I.P. Ivanov*}

INFN - Gruppo Collegato di Cosenza, I-87036 Rende, Italy

and Sobolev Institute of Mathematics, RU-630090, Novosibirsk, Russia

E-mail: igivanovecs.infn.it

\begin{abstract}
We analyze $J^{P C}=3^{--}$meson production in diffractive DIS within the $k_{t}$-factorization approach, with a particular attention paid to the $\rho_{3}(1690)$ meson. We find $\rho_{3}(1690)$ production cross section is of the same order of magnitude as the $\rho^{\prime \prime}(1700)$ production cross section. Studying $\sigma_{L}$ and $\sigma_{T}$ separately, we observe domination of $\rho_{3}$ in $\sigma_{L}$ and domination of $\rho(1700)$ in $\sigma_{T}$ and offer an explanation of this behavior in terms of Clebsch-Gordan coefficients. We also find very strong contributions, sometimes even domination, of the $s$-channel helicity violating amplitudes. The typical color dipole sizes probed in $\rho_{3}$ production are larger than those in the ground state $\rho$ production. All the conclusions about the relative behavior of $\rho_{3}(1690)$ and $\rho(1700)$ mesons are numerically stable against variations of input parameters.
\end{abstract}

International Workshop on Diffraction in High-Energy Physics -DIFFRACTION 2006 September 5-10 2006

Adamantas, Milos island, Greece

${ }^{*}$ Speaker. 


\section{Introduction}

Thanks to excellent operation of HERA, diffractive production of vector mesons (VM) in DIS $\gamma^{*} p \rightarrow V p(V=\rho, \phi, J / \psi$ etc. $)$ has become a very active field of research in the last decade (see recent review [1] and references therein). Experimental efforts so far were focused on the production of the ground state mesons, while the published data on diffractive production of excited states are very scarce. The only case studied in detail was the radially excited charmonium, $\psi(2 S)$, production, [2], while for the production of excited $\rho$ mesons one can consult only the fixed target experiments publications, [3, 4, 5].

Theoretical calculations show, however, that detailed study of excited mesons in diffraction should be a very rewarding enterprise. Production of light excited mesons ( $\rho^{\prime}$ mesons, etc) is especially interesting. First, in the soft regime one can probe the dipole cross section at larger dipole sizes than in ground state production, [6], which might help understand the phenomenon of saturation. Then, diffractive production of $\rho^{\prime}$ can lead to resolution of the long standing puzzle of the radial/orbital excitation assignment to the $\rho(1450)$ and $\rho(1700)$ mesons, see [7]. Finally, the remarkable property of orbitally excited mesons is that their $s$-channel helicity conserving (SCHC) production amplitudes suffer an extra suppression in comparison with ground state production, while the helicity violating amplitudes (SCHNC) do not, [8, 9]. This hints at the increased importance of helicity violation in production of mesons with large orbital excitations.

We report here calculations of the diffractive DIS production of spin-excited mesons, in particular, the spin-3 meson $\rho_{3}(1690)$. We use the $k_{t}$-factorization approach and adapt the formalism of [8] to high-spin mesons. For numerical calculations we use the fits to the unintegrated gluon density developed in [10]. Details on these calculations can be found in [11].

\section{Spin-3 meson production: analytical results}

We use the usual notation for kinematical variables. $Q^{2}$ is the photon's virtuality, $W$ is the total center-of-mass energy of the $\gamma^{*} p$ collision. The momentum transfer from proton to photon is denoted by $\Delta_{\mu}$ and at high energies is almost purely transverse: $-\Delta^{2}=|t| \approx\left|t^{\prime}\right|=\vec{\Delta}^{2}$.

Diffractive production of meson $V$ with mass $m_{V}$ can be treated in the lowest Fock state approximation as production of the corresponding $q \bar{q}$ pair of invariant mass $M \neq m_{V}$, which is then projected, at the amplitude level, onto the final state. Within the leading $\log \frac{1}{x}$ accuracy the higher Fock states are reabsorbed into the evolution of the unintegrated gluon density (or color dipole cross section). A typical diagram to be calculated (see [1]) contains the valence quark loop, with integration over the quark transverse momentum $\vec{k}$ and its fraction of photon's lightcone momentum $z$, and the uppermost gluon loop, with the integration over transverse momentum $\vec{\kappa}$. A convenient choice is to assign momentum $\vec{k}+z \vec{\Delta}$ to the quark and $-\vec{k}+(1-z) \vec{\Delta}$ to the antiquark, which ensures that even at non-zero $\vec{\Delta}$ the $q \bar{q}$ invariant mass is $M^{2}=\left(m^{2}+\vec{k}^{2}\right) / z(1-z)$. It is also convenient to consider the relative $q \bar{q}$ momentum $p^{\mu} \equiv\left(k_{q}-k_{\bar{q}}\right)^{\mu} / 2$ in the $q \bar{q}$ pair rest frame, where it reduces to the 3-momentum $\mathbf{p}=\left(\vec{k}, k_{z}\right)$ with $k_{z} \equiv(2 z-1) M / 2$. Such 3-dimensional vectors, which always refer to the $q \bar{q}$ rest frame, will be given in bold. 
A generic form of the helicity amplitudes $\gamma^{*}\left(\lambda_{\gamma}\right) \rightarrow V_{3}\left(\lambda_{3}\right)$ is

$$
\operatorname{Im} A_{\lambda_{3} ; \lambda_{\gamma}}=W^{2} \frac{c_{V} \sqrt{4 \pi \alpha_{e m}}}{4 \pi^{2}} \int \frac{d z d^{2} \vec{k}}{z(1-z)} \int \frac{d^{2} \vec{\kappa}}{\vec{\kappa}^{4}} \alpha_{s} \mathscr{F}\left(x_{1}, x_{2}, \vec{\kappa}, \vec{\Delta}\right) \cdot I_{\lambda_{3} ; \lambda_{\gamma}}^{(3)} \cdot \psi_{3}\left(\mathbf{p}^{2}\right)
$$

Here $c_{V}$ is the flavor factor, $\mathscr{F}\left(x_{1}, x_{2}, \vec{\kappa}, \vec{\Delta}\right)$ is the skewed unintegrated gluon distribution.

A spin-3 particle is described with the rank-3 polarization tensor $T^{\mu v \rho}$, which must be symmetric and traceless in any pair of Lorentz indices. Its coupling to the $q \bar{q}$ pair can be expressed via the coupling of the $S$-wave vector meson:

$$
\bar{u} \mathscr{C}^{\mu v \rho} u \cdot T_{\mu v \rho}=\bar{u} \mathscr{S}^{\mu} p^{v} p^{\rho} u \cdot T_{\mu v \rho} \equiv \bar{u} \mathscr{S}^{\mu} u \cdot \tau_{\mu}
$$

where $\tau_{\mu} \equiv T_{\mu v \rho} p^{v} p^{\rho}$ plays the role of effective polarization vector. This allows one to construct the integrands $I_{\lambda_{3} ; \lambda_{\gamma}}^{(3)}$ for spin-3 meson production directly from the known integrands $I_{\lambda_{s} ; \lambda_{\gamma}}^{S}$ for the $S$-wave vector meson production.

The shape of the radial wave function of the spin-3 meson is governed by the angular momentum $L=2$. Note that spin-3 meson $(L=2, S=1 \rightarrow J=3)$ is a spin-orbital splitting partner of the $D$-wave vector meson $(L=2, S=1 \rightarrow J=1)$, and according to the potential models one can assume that their radial distribution functions are proportional to each other. The normalization condition then gives $\psi_{3}\left(\mathbf{p}^{2}\right)=\sqrt{15} \psi_{D}\left(\mathbf{p}^{2}\right)$, which will be useful for comparison of spin-3 and $D$-wave vector meson production.

The above expressions can be integrated numerically, but some key issues can be understood analytically. We first show why production of higher spin meson must be non-zero at all. Consider a simpler problem: transformation of a purely $S$-wave $q \bar{q}$ state into the $D$-wave $q \bar{q}$ state just in vacuum. One expects the amplitude of this transition to be zero due to the $S$-wave/ $D$-wave orthogonality. Indeed, one finds

$$
\mathscr{M} \propto \int \frac{d z}{z(1-z)} d^{2} \vec{k} \psi_{D}^{*}\left(\mathbf{p}^{2}\right) \psi_{S}\left(\mathbf{p}^{2}\right)\left(2 k_{z}^{2}-\vec{k}^{2}\right)=\int \frac{4}{M} d^{3} \mathbf{p} \psi_{D}^{*}\left(\mathbf{p}^{2}\right) \psi_{S}\left(\mathbf{p}^{2}\right)\left(2 k_{z}^{2}-\vec{k}^{2}\right)=0 .
$$

The presence of the quadrupole combination $2 k_{z}^{2}-\vec{k}^{2}$ makes the amplitude zero, as long as all other factors under the integral are spherically symmetric.

In diffraction, such a transition is induced by the Pomeron (the diffraction operator), and one has

$\psi_{S}\left(p^{2}\right) \rightarrow \psi_{S}\left(k_{z}^{2}+\left(\vec{k}_{\perp}+\vec{\kappa}\right)^{2}\right)+\psi_{S}\left(k_{z}^{2}+\left(\vec{k}_{\perp}-\vec{\kappa}\right)^{2}\right)-2 \psi_{S}\left(k_{z}^{2}+\vec{k}_{\perp}^{2}\right) \approx 2 \vec{\kappa}^{2}\left[\psi_{S}^{\prime}\left(p^{2}\right)+\vec{k}_{\perp}^{2} \psi_{S}^{\prime \prime}\left(p^{2}\right)\right]$,

which lacks spherical symmetry. Therefore

$$
\int d^{3} p\left[\psi_{S}^{\prime}+\vec{k}_{\perp}^{2} \psi_{S}^{\prime \prime}\right] \psi_{D} \cdot\left(2 k_{z}^{2}-\vec{k}_{\perp}^{2}\right) \neq 0 .
$$

This is a natural result. The collision process involves a preferred direction: that of the proton's momentum in the vector meson rest frame. The transverse and longitudinal dynamics of the quark loop now differ, and this leads, in particular, to circulation of purely transverse momentum $\vec{\kappa}$ in the loop, which breaks the spherical symmetry.

The second issue that can be understood analytically is the relative production rate of the spin-orbital partners, $V_{D}$ and $V_{3}$, in the high- $Q^{2}$ and high- $m_{V}^{2}$ limit in the forward case. In this 
case $\sigma\left(V_{3}\right), \sigma\left(V_{D}\right) \ll \sigma(V)$, but $\sigma\left(V_{3}\right)$ and $\sigma\left(V_{D}\right)$ are comparable. The most remarkable result one obtains in this limit is on the longitudinal to transverse ratios $R_{L T} \equiv\left(\sigma_{L} / \sigma_{T}\right) \cdot\left(m_{V}^{2} / Q^{2}\right)$. For example, for $Q^{2}=m_{V}^{2}$ the results for ground state vector meson, the $D$-wave excitation and the spin-3 meson are: $R_{L T}^{1 S}=1, R_{L T}^{D}=9 / 289, R_{L T}^{3}=6$, which differ by orders of magnitude. Such a dramatic difference originates from $\sigma_{L}^{3} \gg \sigma_{L}^{D}, \quad \sigma_{T}^{3} \ll \sigma_{T}^{D}$. The cause of this "mirror" behavior can be traced back to Clebsch-Gordan coefficients, i.e. to the spin-orbital structures of $V_{3}$ and $V_{D}$. Consider the integrands that enter the production amplitudes of +1 states of $V_{3}$ and $V_{D}$ :

$$
\begin{aligned}
& I_{+1}^{3}=\frac{1}{\sqrt{15}}\left[\left(2 k_{z}^{2}-\vec{k}_{\perp}^{2}\right) I_{+}^{S}+4 k_{z} k_{+}^{*} I_{0}^{S}+\left(k_{+}^{*}\right)^{2} I_{-}^{S}\right] ; \\
& I_{+1}^{D}=-\frac{1}{2}\left[\left(2 k_{z}^{2}-\vec{k}_{\perp}^{2}\right) I_{+}^{S}-6 k_{z} k_{+}^{*} I_{0}^{S}+6\left(k_{+}^{*}\right)^{2} I_{-}^{S}\right] .
\end{aligned}
$$

The second terms have opposite signs for $V_{3}$ and $V_{D}$, so that the interference patterns are distinctly different. It is this difference that leads to $\sigma_{T}^{3} \ll \sigma_{T}^{D}$. The opposite phenomenon happens for the longitudinal cross sections. Since these phenomena stem from the very definition of spin-3 and $D$-wave vector meson polarization states, one can hope that the property $R_{L T}^{3} \gg R_{L T}^{D}$ is a modelindependent prediction. It would be interesting to verify it in other theoretical approaches and to test it experimentally.

\section{Spin-3 meson production: numerical results}

We applied the above results to study numerically the production of $\rho_{3}(1690)$ meson. In order to integrate (2.1) numerically, one needs to specify models for the unintegrated gluon density and the meson wave function. We related the skewed unintegrated gluon density with non-zero momentum transfer to the forward unintegrated gluon density, and for the latter we used parametrizations borrowed from [10]. Note that the $k_{t}$-factorization approach itself does not require $Q^{2}$ to be large, and in the soft region, $Q^{2} \lesssim 1 \mathrm{GeV}^{2}$, the words "unintegrated gluon density" should be understood simply as an appropriately normalized Fourier transform of the dipole cross section.

As for the radial wave function, we choose the simple Gaussian Ansatz WF and, additionally, assumed proportionality between $\psi_{3}\left(\mathbf{p}^{2}\right)$ and $\psi_{D}\left(\mathbf{p}^{2}\right)$. The size of the wave function has been extracted from $\Gamma\left(\rho(1700) \rightarrow e^{+} e^{-}\right)$. Unfortunately, this width is known very poorly, to factor of $\sim 5$, and this error propagates in our calculations. So, our numerical results for the absolute values of the cross sections are only to indicate the order of magnitude. However, the accuracy for the observables that depend on the ratios of the amplitudes as well as for the relative production rates of $\rho_{3}$ and $\rho^{\prime \prime}(1700)$ is much better, within factor of 2 .

Fig. 1 shows the ratios of the excited to ground state meson cross sections $\sigma\left(\rho_{3}\right) / \sigma\left(\rho_{1 S}\right)$ and $\sigma\left(\rho_{D}\right) / \sigma\left(\rho_{1 S}\right)$. Both ratios are an order of magnitude smaller than unity, and the $\rho_{D}$ cross section is noticeably larger than that of $\rho_{3}$, especially at $Q^{2} \sim 1 \mathrm{GeV}^{2}$. At larger $Q^{2}$, the ratio $\sigma\left(\rho_{D}\right) / \sigma\left(\rho_{3}\right) \sim$ 2. Thus, if one intends to extract $\rho(1700)$ properties from diffractively produced multipion states around invariant mass $\sim 1700 \mathrm{MeV}$, one cannot neglect contamination by the $\rho_{3}$ state.

Difference between $\rho_{D}$ and $\rho_{3}$ is better seen if one studies separately longitudinal and transverse cross sections, shown in Fig. 2. Here, the solid and dash-dotted lines represent the $\rho_{3}$ and $\rho_{D}$ cross sections, respectively. In the case of $\rho_{3}$ we showed also with the dashed lines the contributions of the SCHC amplitudes only. One clearly sees the domination of helicity violating 


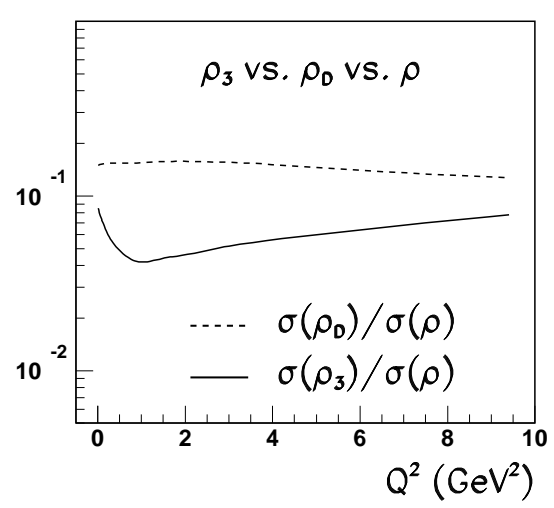

Figure 1: Predictions for the ratios of $\rho_{3}$ (solid line) and $\rho_{D}$ (dashed line) to $\rho$ production cross sections as a function of $Q^{2}$.
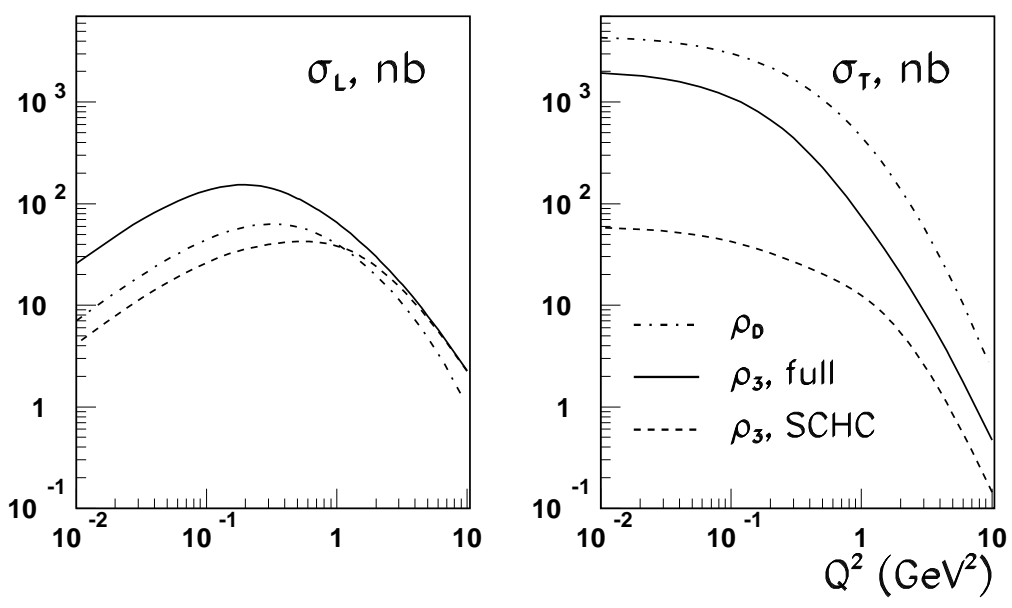

Figure 2: Predictions for the longitudinal (left plot) and transverse (right plot) cross sections of $\rho_{3}$ (solid lines) and $\rho_{D}$ (dash-dotted lines) production. The contribution to $\rho_{3}$ from SCHC amplitudes only is shown with dashed lines.

amplitudes at small $Q^{2}$ in $\rho_{3}$ production. One can even state, on the basis of our calculations, that $\rho_{3}$ production at small $Q^{2}$ probes diffraction in the regime of strong s-channel helicity violation. In the case of longitudinal cross section, the contribution of SCHNC transitions becomes small at $Q^{2}>1 \mathrm{GeV}^{2}$, since all such amplitudes are of higher twist. Helicity violation remains strong for transverse photons even at large $Q^{2}$.

As mentioned above, in the case of $D$-wave vector mesons one expects suppression of $\sigma_{L}$ but not in $\sigma_{T}$. Indeed, our calculations show the domination of the $\rho_{3}$ over $\rho_{D}$ in $\sigma_{L}$ at small $Q^{2} \lesssim 1$ $\mathrm{GeV}^{2}$, while in $\sigma_{T}$ the $\rho_{D}$ cross section is noticeably larger than $\rho_{3}$ everywhere. This is in a qualitative agreement with expectations.

It is also interesting to note that $\rho_{3}$ photoproduction is dominated by significantly larger color dipoles, by a factor of $\approx 1.5$, than the $\rho$ production. This is due to both a broader radial wave func- 
tion for $L=2$ state as well as to the strong contribution of double spin flip amplitudes $+1 \rightarrow-1$ and $+1 \rightarrow+3$, which are dominated by soft scattering. This property suggests $\rho_{3}$ photoproduction as a unique testing grounds of large color dipole dynamics and, possibly, of the saturation phenomenon.

\section{Experimental possibilities}

The OMEGA Collaboration at CERN measured the cross section of diffractive photoproduction of $\rho_{3}(1690)$ (known then as $g(1690)$ meson) via the $a_{2}(1320) \pi$ subsample of the $\eta \pi^{+} \pi^{-}$ diffractive final state events [4]. The cross section of $\gamma p \rightarrow \rho_{3}(1690) p \rightarrow a_{2}(1230) \pi p$ was found to be $97 \pm 28 \pm 21 \mathrm{nb}$, which allows one to roughly estimate the $\rho_{3}$ production cross section as $\sigma\left(\gamma p \rightarrow \rho_{3}(1690) p\right) \sim 200-300 \mathrm{nb}$. This result is about 5-10 times below our photoproduction predictions, which we think is not very bad discrepancy, taking into account expected level of accuracy in the soft region.

The OMEGA Collaboration also measured the photoproduction cross section of $\rho^{\prime}$. The original data were reanalyzed in terms of $\rho(1450)$ and $\rho(1700)$ separately in [12] yielding $\sigma(\rho(1700)) \sim$ $500 \mathrm{nb}$. Thus, our result $\sigma(\rho(1700)) / \sigma\left(\rho_{3}\right) \sim 3$ at the photoproduction limit is roughly consistent with experiment. Finally, comparing the $\rho_{3}$ and the ground state $\rho$ photoproduction cross sections, we note that our result $\sigma_{3} / \sigma_{1 S} \sim 0.1$ is again not very far from experimental value of 0.02-0.03.

Some of the current fixed target experiments, in particular, COMPASS at CERN and E831 at FNAL, have large potential in study of the analysis of this process. Detailed analysis of experimental opportunities is discussed in [11].

\section{Conclusions}

We calculated the cross section of the exclusive production of $J^{P C}=3^{--}$mesons in diffractive DIS within the $k_{t}$-factorization approach. The results were compared with the cross section of the $D$-wave state vector meson of the same quarkonium. We exemplified the general expressions with a detailed numerical study of the $\rho$ system, where the $\rho_{3}(1690)$ state is almost degenerate with the $\rho(1700)$ meson, whose structure is arguably dominated by the $q \bar{q}$ pair in the $D$ wave.

The absolute values of the cross sections suffer from uncertainties of the input parameters, in particular, of the $\rho_{D} \rightarrow e^{+} e^{-}$decay width, and we can be sure only in the order of magnitude of these results. However, in what concerns the relative production rates of $\rho_{3}$ and $\rho_{D}$, our conclusions are much more certain. Our results allow us to formulate the following predictions, which are stable against variations of the model parameters:

- In typical HERA kinematics, the ratio of production cross sections taken at equal $Q^{2}$ is $\sigma\left(\rho_{D}\right) / \sigma\left(\rho_{3}\right) \approx 3-5$ at small $Q^{2}$, decreasing to $\approx 2$ at larger $Q^{2}$. Thus, when extracting the properties of $\rho$ (1700) from multipion final states, one cannot simply neglect the $\rho_{3}$ contribution.

- $\rho_{3}$ and $\rho_{D}$ show completely different patterns in $\sigma_{L^{-}} \sigma_{T}$ decomposition: $\rho_{3}$ dominates in the longitudinal cross section, while $\rho_{D}$ dominates in the transverse cross section. The ratios $R=\sigma_{L} / \sigma_{T}$ for $\rho_{3}$ and $\rho_{D}$ differ by more than one order of magnitude. 
- The role of $s$-channel helicity violating amplitudes is extremely important, especially in the transverse cross section and at small $Q^{2}$. Production of $\rho_{3}$ offers an interesting possibility to study diffraction in the regime of strong $s$-channel helicity violation.

We find no surprise in numerical stability of the first two conclusions, since they are essentially driven by very basic relations: similarity of radial wave functions for $\rho_{3}$ and $\rho_{D}$, the spin-angular composition of these mesons, and quadrupole suppression of the leading contributions in SCHC amplitudes.

The work of I.P.I. was supported by the INFN Fellowship, and partly by INTAS and grants RFBR 05-02-16211 and NSh-2339.2003.2.

\section{References}

[1] I. P. Ivanov, N. N. Nikolaev and A. A. Savin, Diffractive vector meson production at HERA: From soft to hard QCD, Phys. Elem. Part. Atom. Nucl. 66 (2006) 1 [hep-th/ 0501034$].$

[2] C. Adloff et al., Diffractive photoproduction of psi(2S) mesons at HERA, Phys. Lett. B 541 (2002) 251 [hep-ex/0205107].

[3] D. Aston et al., Observation of the rho-prime (1600) in the channel gamma $p \rightarrow$ pi+ pi-p., Phys. Lett. B 92 (1980) 215; The Reaction Gamma $P \rightarrow P$ Pi+ Pi- Pi+ Pi- For Photon Energies From 25-GeV To 70-GeV, Nucl. Phys. B 189 (1981) 15; M. Atkinson et al., The Decay Of The Rho-Prime (1600) Into Pi+ Pi-PiO PiO, Phys. Lett. B 108 (1982) 55; The Rho-Prime (1600) In The Reaction Gamma P $\rightarrow$ Pi+ Pi- PiO PiO P At Photon Energies Of 20-GeV To 70-GeV, Z. Phys. C 26 (1985) 499.

[4] M. Atkinson et al., Study Of Eta Pi+ Pi-States In The Rho-Prime (1600) Mass Region Photoproduced In The Reaction Gamma P $\rightarrow$ Eta Pi+ Pi- P At Photon Energies Of 20-GeV To 70-GeV, Z. Phys. C 30 (1986) 531.

[5] P. Lebrun [E687 Collaboration], Recent results from Fermilab E687, in proceedings of Hadron 97, Upton, NY, 25-30 August 1997.

[6] G. Kulzinger, H. G. Dosch and H. J. Pirner, Diffractive photo- and leptoproduction of vector mesons rho, rho-prime and rho-prime-prime, Eur. Phys. J. C 7 (1999) 73 [hep-ph/9806352].

[7] D. V. Bugg, Four sorts of meson, Phys. Rept. 397 (2004) 257 [hep-ex/ 0412045 ].

[8] I. P. Ivanov and N. N. Nikolaev, Diffractive $S$ and D wave vector mesons in deep inelastic scattering, JETP Lett. 69 (1999) 294 [hep-ph/9901267].

[9] F. Caporale, I. P. Ivanov, Production of orbitally excited vector mesons in diffractive DIS, Phys. Lett. B 622 (2005) 55 [hep-ph/ 0505266].

[10] I. P. Ivanov and N. N. Nikolaev, Anatomy of the differential gluon structure function of the proton from the experimental data on $F(2 p)\left(x, Q^{* * 2}\right)$, Phys. Rev. D 65 (2002) 054004 [hep-ph/ 0004206 ].

[11] F. Caporale, I. P. Ivanov, Production of spin-3 mesons in diffractive DIS, Eur. Phys. J. C 44 (2005) 505 [hep-ph/0504139].

[12] A. Donnachie and H. Mirzaie, Evidence for two rho-prime (1600) resonances, Z. Phys. C 33 (1987) 407. 